\title{
Optimum PSK Signal Mapping for Multi-Phase Binary-CDMA Systems
}

\author{
Yeong-Jin Seo and Yong-Hwan Lee \\ School of Electrical Engineering and INMC, Seoul National University \\ Kwanak P. O. Box 34, Seoul, 151-744, Korea \\ e-mail: ylee@snu.ac.kr
}

\begin{abstract}
Although the CDMA system can efficiently support multiple users, may suffer from peak-to-average power ratio (PAPR) increases as the number of users increases. As a result, it needs highly linear power amplifiers with a large back off. Recently, a new CDMA scheme, called binary CDMA (B-CDMA), has been proposed to alleviate this problem by quantizing the envelope of multi-user CDMA signals into a small number of levels, while preserving the advantages of CDMA signaling [1]. The performance of B-CDMA system is mainly determined by the quantization and detection error. The quantization noise can be minimized using the Lloyd-max algorithm [2]. In this paper, the optimum PSK signal is designed to minimize the detection error in multi-phase B-CDMA systems. Finally, the analytic results are verified by computer simulation.
\end{abstract}

\section{INTRODUCTION}

One of major drawbacks of multi-code CDMA systems is high peak-to-average power ratio (PAPR) due to the aggregation of multiple spreading codes. As a result, multicode CDMA transmitters require the use of highly linear power amplifiers with a large back off. Binary CDMA (BCDMA) is a new modulation method that quantizes the signal amplitude into a small number of levels and employs PSK-modulation for transmission with constant envelope [1] Thus, the B-CDMA can alleviate the need of linear power amplifiers, while preserving the advantages of CDMA

This work was supported by Korea Research Foundation. signaling such as the soft capacity and robustness to interference. However, the performance can significantly be affected by the quantization process.

The B-CDMA signal can be generated by various methods including the pulse-width (PW), multi phase (MP) and code selection (CS) methods [1]. The PW B-CDMA signal is obtained by converting the magnitude of multi-level signal into a finite number of pulse width. Thus, the transmission bandwidth of the PW B-CDMA increases as the quantization level increases. In practice, the signal can be quantized into two levels to accommodate the increase of transmission bandwidth. The MP B-CDMA is generated by transforming the signal amplitude into a finite number of PSK signal constellation. The CS B-CDMA is generated by a two-step process. In the first step, the subset of spreading codes is selected to reduce the number of signal levels. In the second step the selected code is modulated using the MP B-CDMA scheme.

Optimum quantization of the signal amplitude can be achieved by using the Lloyd-max algorithm [2]. However the PSK signal constellation has not been optimized analytically for the MP B-CDMA. This is mainly due to the fact that the mean square error of the chip decision error and the symbol error in the PSK system cannot be represented in a simple form. The two signal points having the largest distance after the quantization are PSK-mapped so that they have the largest distance on the PSK signal constellation [3]. For a given PSK signal constellation, the decision region can be determined so as to minimize the Bayes cost criterion [4]. However it may not be optimum because the bit error rate (BER) performance can be more affected by the PSK signal mapping than the decision region. In this paper, we optimize the PSK mapping points of the MP B-CDMA signal in additive white Gaussian noise (AWGN) channel. Since the 


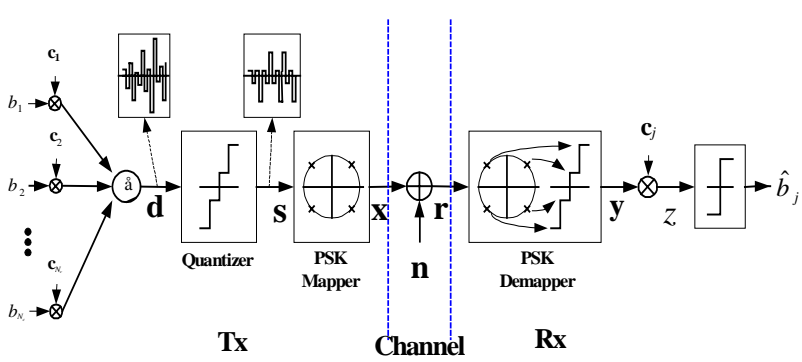

Fig. 1. MP B-CDMA transceiver structure

CS B-CDMA is the same as the MP B-CDMA except the code selection block [5], the analytic design can also be applied to optimum design of the CS B-CDMA signal.

Section 2 describes the structure of the MP B-CDMA system. In Section 3, the noise due to the quantization and chip detection error is analyzed. The PSK mapping points are optimized to minimize the chip detection error using an iterative method. The proposed signal mapping scheme is evaluated using computer simulation in Section 4. Conclusions are summarized in Section 5.

\section{SySTEM MODEL}

In the MP B-CDMA system, the sum of multiple users data is quantized into a finite number of levels and then modulated using a PSK modulation scheme. Fig. 1 depicts the transceiver structure of a baseband-equivalent MP BCDMA system with quantization level 4 , where $b_{i}$ and $\mathbf{c}_{i}$ respectively denote the bit and spreading code of the $i$-th user, and the signal $\mathbf{d}$ denotes the sum of multiple users data, given by

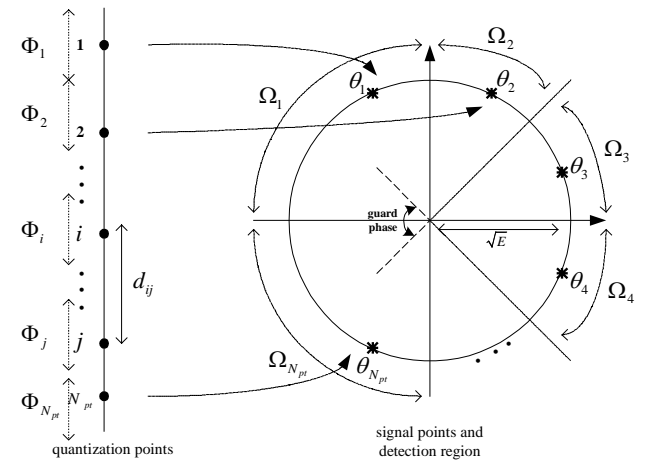

Fig. 2. PSK modulation of MP B-CDMA

$$
\mathbf{d}=\sum_{i=1}^{N_{c}} b_{i} \mathbf{c}_{i}
$$

Here, $N_{c}$ is the total number of users.

The aggregated user signal $\mathbf{d}$ is quantized at the chiplevel. The output of the quantizer can be represented as

$$
\mathbf{s}=f_{q}(\mathbf{d})
$$

where $f_{q}(\mathbf{d})$ denotes the quantization function that maps the signal $\mathbf{d}$ in the quantization region $\Phi_{i}$ onto the signal point $m_{i}$ at the chip-level. Then, the quantized signal $\mathbf{s}$ is PSK mapped as

$$
\mathbf{x}=f_{\text {map }}(\mathbf{s})
$$

where $f_{\text {map }}(\mathbf{s})$ denotes the mapping function that maps the signal $\mathbf{s}$ onto the PSK constellation. Fig. 2. depicts the quantization and PSK-mapping region of the MP B-CDMA system, where $d_{i j}$ denotes the distance between the quantized signal point $i$ and $j, \Omega_{i}$ denotes the detection region of the PSK-modulated signal point $i, N_{p t}$ is the number of signal points after the quantization. Note that the guard phase is required in the MP B-CDMA system to reduce the decision errors between the signal points having the largest distance [3].

The received signal can be written as

$$
\mathbf{r}=\mathbf{x}+\mathbf{n}
$$

where $\mathbf{n}$ denotes the AWGN term. The PSK demodulator transforms the phase information of $\mathbf{r}$ into the magnitude

$$
\mathbf{y}=f^{-1}{ }_{\text {map }}(\mathbf{r})
$$

The demodulated signal $\mathbf{y}$ is despreaded using the spreading code $\mathbf{c}_{j}$ of the $j$-th user to detect the user data $\hat{b}_{j}$ as

$$
\hat{b}_{j}= \begin{cases}1 & , \text { if } \mathbf{y} \cdot \mathbf{c}_{j} \geq 0 \\ 0 & , \text { if } \mathbf{y} \cdot \mathbf{c}_{j}<0\end{cases}
$$

Let $s_{j}, \delta_{j}$ and $e_{j}$ be the signal component, quantization noise and noise due to chip detection error at the $j$-th chip, respectively. Then, the demodulated signal $\mathbf{y}$ at the $j$-th chip can be represented as 


$$
y_{j}=s_{j}+\delta_{j}+e_{j}
$$

\section{OPTIMUM PSK SIGNAL CONSTELLATION FOR MP B-CDMA}

Since there is no correlation between $\delta_{j}$ and $e_{j}$, the variance of $y_{j}$ can be written as

$$
\sigma_{y}^{2}=\sigma_{\delta}^{2}+\sigma_{e}^{2}
$$

where $\sigma_{\delta}{ }^{2}$ and $\sigma_{e}{ }^{2}$ are the variance of $\delta_{j}$ and $e_{j}$, respectively. Assuming that the spreading code of each user has unit power, the variance of $d_{j}$ corresponding to the $j$ th chip of $\mathbf{d}$ is equal to the number of multi-codes, $N_{c}$. The variance of the quantization noise per each chip, $\sigma_{\delta}{ }^{2}$, can be represented as [6]

$$
\sigma_{\delta}^{2}=\sum_{i=1}^{N_{p t}} \int_{\Phi_{i}}\left(x-m_{i}\right)^{2} \frac{1}{\sqrt{2 \pi N_{c}}} \exp \left(-\frac{x^{2}}{2 N_{c}}\right) d x
$$

The optimum $m_{i}$ and $\Phi_{i}$ minimizing $\sigma_{\delta}{ }^{2}$ can be obtained using the Lloyd-max algorithm [2]. The Lloyd-max algorithm can find the optimum quantization level in an iterative manner so as to minimize the quantization noise power. The variance of $e_{j}$ can be calculated as

$$
\sigma_{e}^{2}=\sum_{i=1}^{N_{p t}} \sum_{j=1}^{N_{p t}} p(j \mid i) p_{s}(i) d_{i j}^{2}
$$

where $p_{s}(i)$ is the probability density function (pdf) of signal point $i$ given by

$$
p_{s}(i)=\int_{\Phi_{i}} \frac{1}{\sqrt{2 \pi N_{c}}} \exp \left(-\frac{x^{2}}{2 N_{c}}\right) d x
$$

and $p(j \mid i)$ is the probability that signal point $i$ is misdetected to $j$ [6]

$$
p(j \mid i)=\int_{\Omega_{j}} \int_{0}^{\infty} \frac{1}{2 \pi} e^{-\gamma_{s} \sin ^{2}\left(\theta_{r}-\theta_{i}\right)} R^{-\left(R-\sqrt{2 \gamma_{s}} \cos \left(\theta_{r}-\theta_{i}\right)\right)^{2} / 2} d R d \theta_{r}
$$

Table 1. Mapping phase

\begin{tabular}{|c|c|c|c|}
\hline$E_{b} / N_{o}$ & Conventional & Exhaustive search & Proposed \\
\hline $10 d B$ & $-135^{\circ},-90^{\circ},-45^{\circ}$ & $-120^{\circ},-85^{\circ},-46^{\circ}$ & $-118^{\circ},-75^{\circ},-30^{\circ}$ \\
\hline $11 d B$ & $-135^{\circ},-90^{\circ},-45^{\circ}$ & $-125^{\circ},-91^{\circ},-45^{\circ}$ & $-124^{\circ},-80^{\circ},-34^{\circ}$ \\
\hline $12 d B$ & $-135^{\circ},-90^{\circ},-45^{\circ}$ & $-127^{\circ},-85^{\circ},-46^{\circ}$ & $-129^{\circ},-84^{\circ},-38^{\circ}$ \\
\hline
\end{tabular}

Here, $\gamma_{s}$ is the chip energy to noise power ratio ( i.e., $E_{c} / N_{o}$ ) and $\theta_{i}$ is the phase of signal point $i$. Provided that $\gamma_{s}$ is high enough, most of chip detection errors are associated with the decision to the adjacent signal points. Thus the variance of the chip detection error can be approximated as

$$
\sigma_{e}^{2}=\sum_{i=1}^{N_{p t}} \sum_{j=i_{-}, i_{+}} p(j \mid i) p_{s}(i) d_{i j}^{2}
$$

where $i_{-}$and $i_{+}$denote the adjacent signal points of signal point $i$.

Since (12) involves nonlinear functions, we approximate $p(j \mid i)$ for ease of mathematical analysis. It can be shown that

$$
\begin{aligned}
& p(j \mid i)=\int_{\Omega_{j}}\left[\frac{1}{2 \pi} e^{-\gamma_{s} \sin ^{2}\left(\theta_{r}\right)} \int_{0}^{\infty} R e^{-\left(R-\sqrt{2 \gamma_{s}} \cos \left(\theta_{r}\right)\right)^{2} / 2} d R\right] d \theta_{r} \\
& =\int_{\Omega_{j}}\left[\frac{1}{2 \pi}\left\{\begin{array}{c}
e^{-\gamma_{s}}+e^{-\gamma_{s} \sin ^{2}\left(\theta_{r}\right)} \times \\
\sqrt{\gamma_{s} \pi} \cos \left(\theta_{r}\right) \operatorname{erf}\left(-\sqrt{\gamma_{s}} \cos \left(\theta_{r}\right)\right)
\end{array}\right\}\right] d \theta_{r}
\end{aligned}
$$

Since the first term in (14) can be ignored at high $E_{b} / N_{0}$, (14) can be approximated as

$$
\begin{gathered}
p(j \mid i) \approx \int_{\Omega_{j}} \frac{1}{2 \pi} e^{-\gamma_{s} \sin ^{2}\left(\theta_{r}\right)} \sqrt{\gamma_{s} \pi} \\
\cos \left(\theta_{r}\right) \operatorname{erfc}\left(-\sqrt{\gamma_{s}} \cos \left(\theta_{r}\right)\right) d \theta_{r} \\
=\int_{\Omega_{j}} \frac{1}{2 \pi} e^{-\gamma_{s} \theta_{r}^{2}}\left[\begin{array}{l}
e^{-\gamma_{s}\left(\sin ^{2}\left(\theta_{r}\right)-\theta_{r}^{2}\right)} \times \\
\sqrt{\gamma_{s} \pi} \cos \left(\theta_{r}\right) \operatorname{erfc}\left(-\sqrt{\gamma_{s}} \cos \left(\theta_{r}\right)\right)
\end{array}\right] d \theta_{r}
\end{gathered}
$$

For a small $\theta_{r}, \quad p(j \mid i)$ can further be approximated as 


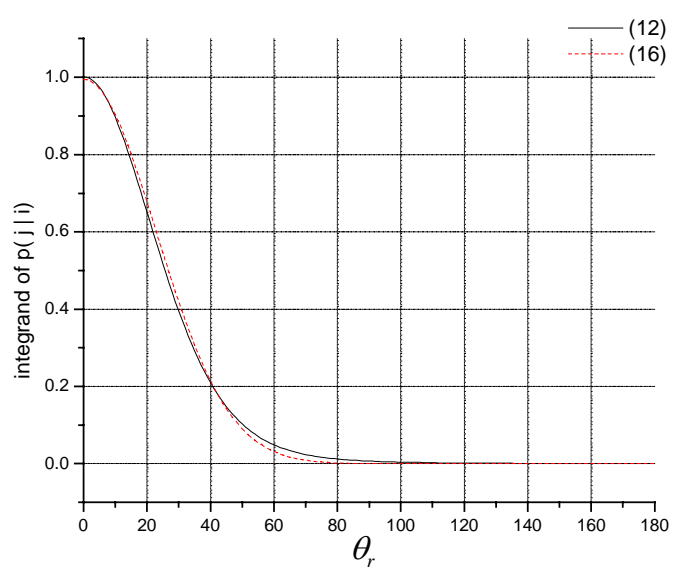

Fig. 3. Approximation of $p(j \mid i)$

$$
p(j \mid i) \approx \int_{\Omega_{j}} \frac{1}{2 \pi} e^{-\gamma_{s} \theta_{r}^{2}} \sqrt{\gamma_{s} \pi} \operatorname{erfc}\left(-\sqrt{\gamma_{s}}\right) d \theta_{r}
$$

As $\theta_{r}$ increases, the difference between the integrand of (12) and (16) increases. However the difference between the two integrals is negligible. Fig. 3 compares the integrand of (12) and (16) when $E_{b} / N_{0}=11 d B, N_{c}=32, \theta_{i}=0^{\circ}$ and spreading factor $N_{S F}=128$. Numerical results indicate that the approximated $p(j \mid i)$ is quite valid in nominal operating condition.

Let $\Omega_{j}$ be the misdetection region of signal point $i$ to $j$ and the corresponding range be from $\omega_{j}$ to $\omega_{j}{ }^{\prime}$, in (16) where, $\omega_{j}{ }^{\prime}=\omega_{j+1}$ and $\omega_{N_{p t}+1}^{\prime}=\omega_{1}$. Because integrand in (16) decreases abruptly as $\theta_{r}$ increases and is not periodic, $\omega_{j}{ }^{\prime}$ can be set to be infinite. Thus, the variance of $e_{j}$ can be written as

$$
\begin{gathered}
\sigma_{e}^{2}=\sum_{i=1}^{N_{p t}} \sum_{j=i_{-}, i_{+}}\left(\int_{\omega_{j}}^{\infty} \frac{1}{2 \pi} e^{-\gamma_{s} \theta_{r}^{2}} \sqrt{\gamma_{s} \pi} \operatorname{erfc}\left(-\sqrt{\gamma_{s}}\right) d \theta_{r}\right) \times \\
\left(\int_{\Phi_{i}} \frac{1}{\sqrt{2 \pi N_{c}}} \exp \left(-\frac{x^{2}}{2 N_{c}}\right) d x\right) d_{i j}{ }^{2}
\end{gathered}
$$

We can find the optimum PSK mapping points $\left(\theta_{1}, \theta_{2}, \cdots, \theta_{N_{p t}}\right)$ that minimize $\sigma_{e}^{2}$ using an iterative method. The optimum phase can be found by

$$
\frac{\partial \sigma_{e}^{2}}{\partial \theta_{i}}=0
$$

It can be shown that $\theta_{i}$ is represented as a function of $\theta_{j}$

$$
\theta_{i}=f_{i}\left(\theta_{j}\right) \quad, \quad \forall j \neq i
$$

It can easily be shown that $\sigma_{e}^{2}$ is a convex function of $\theta_{i}$ and it has a unique global minimum. We can find $\theta_{i}$ using an iterative method with an arbitrary initial value. Note that the optimum $\theta_{i}$ is a the function of $E_{b} / N_{0}$. This implies that the optimum PSK mapping points are associated with the value of $E_{b} / N_{0}$.

\section{Performance evaluation}

To verify the performance improvement, we evaluate the performance of the MP B-CDMA system with $N_{p t}=7$ (i.e. 8-PSK) and one guard phase in AWGN channel using computer simulation. The MP B-CDMA uses an extended PN sequence with $N_{S F}=128$ and $N_{c}=32$ as the spreading code.

From (18), the optimum phase $\theta_{i}$ is determined by

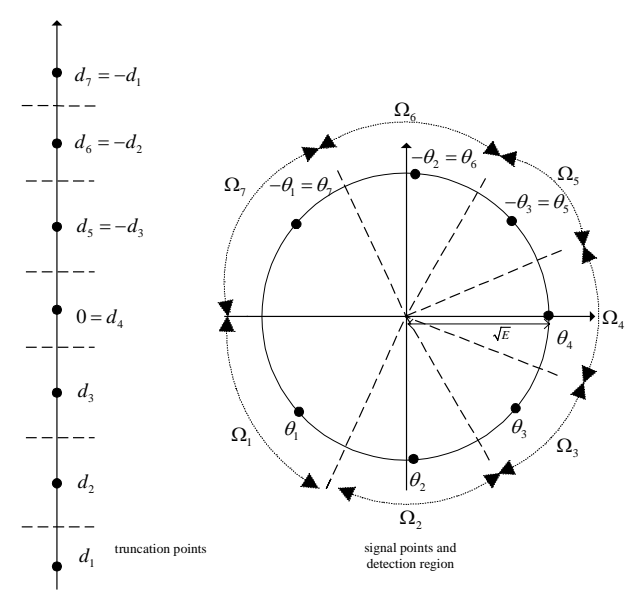

Fig. 4. Proposed 8-PSK signal constellation of the MP B-CDMA 


$$
\begin{aligned}
& \theta_{1}=\frac{1}{3 \gamma_{s}}\left(\sqrt{\left.\frac{-\theta_{2} \gamma_{s}-4 \pi \gamma_{s}-2 \sqrt{\gamma_{s}} \times}{3 \ln \left(\frac{4 d_{17}{ }^{2} p_{s}(1)}{2 d_{12}{ }^{2} p_{s}(1)+2 d_{12}{ }^{2} p_{s}(2)}\right)+\theta_{2}{ }^{2} \gamma_{s}+2 \theta_{2} \pi \gamma_{s}+\pi^{2} \gamma_{s}}\right)}\right. \\
& \theta_{2}=-\frac{4 \log \left(\frac{2 d_{23}{ }^{2} p_{s}(2)+2 d_{23}{ }^{2} p_{s}(3)}{2 d_{12}{ }^{2} p_{s}(1)+2 d_{12}{ }^{2} p_{s}(2)}\right)-\theta_{1}^{2} \gamma_{s}+\theta_{3}^{2} \gamma_{s}}{2\left(\theta_{1}-\theta_{3}\right) \gamma_{s}} \\
& \theta_{3}=\frac{4 \log \left(\frac{2 d_{34}{ }^{2} p_{s}(3)+2 d_{34}{ }^{2} p_{s}(4)}{2 d_{32}{ }^{2} p_{s}(2)+2 d_{23}{ }^{2} p_{s}(3)}\right)+\theta_{2}{ }^{2} \gamma_{s}}{2 \theta_{2} \gamma_{s}}
\end{aligned}
$$

The proposed PSK mapping points $\left\{\hat{\theta}_{1}, \hat{\theta}_{2}, \hat{\theta}_{3}\right\}$ can be found by iteratively searching (20). Conventional PSK mapping points have an equal distance on the signal constellation with one guard phase, that is, $\theta_{1}=-135^{\circ}, \theta_{2}=-90^{\circ}, \theta_{3}=-45^{\circ}$. Alternatively, the optimum PSK points can also be found by exhaustive search.

Table 1 summarizes the conventional, exhaustively searched optimum and proposed PSK mapping points. The proposed point $\hat{\theta}_{1}$ is similar to exhaustively searched optimum point, but $\hat{\theta}_{2}$ and $\hat{\theta}_{3}$ are quite different from the optimum points. Fig. 5 depicts the BER performance of MP B-CDMA with these mapping points. It can be seen that the proposed mapping points can provide the BER performance comparable to the exhaustively searched optimum ones, although $\hat{\theta}_{2}$ and $\hat{\theta}_{3}$ are different from the optimum points. This may imply that $\hat{\theta}_{1}$ with the largest quantization level

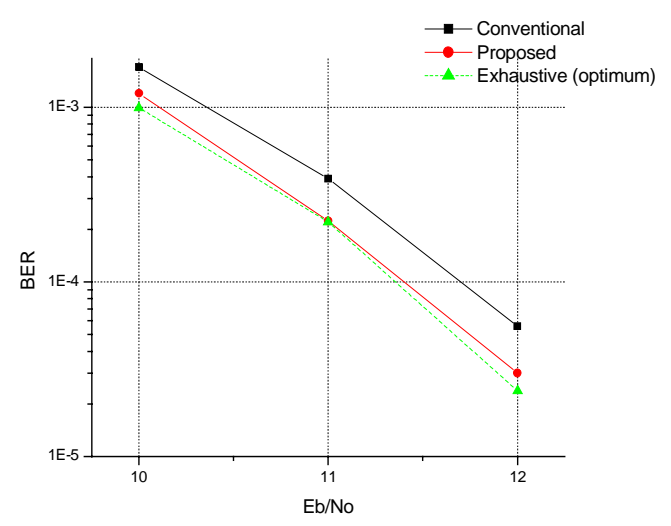

Fig. 5. BER performance due to different phase mapping is the dominant factor on the BER performance.

Fig. 6 depicts the performance of the MP B-CDMA system with the mapping points optimized for each $E_{b} / N_{0}$. It can be seen that the mapping points optimized under a nominal $E_{b} / N_{0}$ condition can be used for certain variation of $E_{b} / N_{0}$ without noticeable performance variation.

\section{Conclusion}

In this paper, we have analytically determined the optimum PSK mapping points of the MP B-CDMA in AWGN channel. The PSK mapping point is optimized by minimizing the mean square error due to chip detection error. The optimum mapping points and obtained using an iterative method using an analytic expression. The simulation results show that the proposed signal constellation can provide near optimum performance, yielding a BER performance gain of about $0.4 d B$ over the conventional one.

\section{REFERENCE}

[1] H. S. An, S. M. Ryu and S. W. Na, "Introduction to Binary CDMA,” Proc. of JCCI 2002, Apr. 2002.

[2] T. M. Cover, Information Theory, Wiley-Interscience, 1991.

[3] E. K. Hong, M. G. Ahn, W. M. Lee and S. M. Ryu, "Design of Signal Constellation for MP/CDMA," Proc. of JCCI 2001, Apr. 2001.

[4] W. M. Lee, E. K. Hong and Y. S. Park, "Design of Optimum Threshold for Chip-Level Multi Phased MCCDMA System with Nonlinear Process," North-East Asia IT Symposium

[5] S. P. Kim, M. J. Kim and H. S. An and S. M. Ryu, "A Constant Amplitude Coding for CS-CDMA System," Proc. of JCCI 2002, Apr. 2002.

[6] J. Y. Ko and Y. H. Lee, "Performance Analysis of Binary-CDMA Transceivers in AWGN Channel," JCCI 2003. Ch. VI-C-1, pp. 1-4. Apr. 2003 